Conclusion $\mathrm{PHH}$ remains to be one of the most severe complications of IVH. In this small group of patients, high $\mathrm{CO}_{2}$ levels, hypotension seem to be important risk factors.

\section{THE EFFECT OF HEAD MOTION ON DATA OUALITY FOR NEONATAL DTI}

doi:10.1136/archdischild-2012-302724.1076

${ }^{1,2} \mathrm{~A}$ Plaisier, ${ }^{1,2} \mathrm{AM}$ Heemskerk, ${ }^{1} \mathrm{KMM}$ Reiss, ${ }^{2} \mathrm{MH}$ Lequin, ${ }^{3} \mathrm{~A}$ Leemans, ${ }^{1,2} \mathrm{~J}$ Dudink. ${ }^{1}$ Neonatology; ${ }^{2}$ Pediatric Radiology, Erasmus MC - Sophia, Rotterdam; ${ }^{3} /$ mage Sciences Institute, University Medical Center Utrecht, Utrecht, The Netherlands

Background and Aims Diffusion Tensor Imaging (DTI) has become valuable for quantitative evaluation of white matter maturation in preterm infants. Because of the occurrence of head movement, gathering good quality data is challenging in neonatal neuroimaging. This is especially of concern for DTI, where motion can result in severe signal drop-out and therefore miscalculation of DTI parameters if data outliers are not handled correctly. This study was aimed to quantify the occurrence of motion artefacts in neonatal DTI and to evaluate different methods for tensor estimation.

Methods We prospectively collected DTI data of 27 preterm infants that were scanned at 30 weeks gestational age. DTI data was acquired in 25 directions. Percentage outliers per slice was calculated. With Explore DTI, we assessed the effect of motion artefacts on tensor estimation using different methods.

Results $60 \%$ of subjects had slightly corrupted data ( $>15$ slices with $>30 \%$ outliers) of which $40 \%$ had severely corrupted data $(>10$ slices with $>50 \%$ outliers). Corrupted data resulted in erroneous DTI parameters. This was especially true for the tensor estimation (ordinary least squares) typically performed by vendors and popular DTI software. More advanced tensor estimations showed more reliable data.

Conclusions Motion artefacts are a major problem in neonatal DTI as it can compromise accurate calculation of DTI parameters. These results press the need for careful data inclusion and the use of reliable methods for tensor estimation. Targeted acquisition, processing and quality assessment is needed in this population to obtain reliable evaluation of white matter maturation.

\section{SEVERE NEONATAL BRAIN INJURY IN VERY LOW BIRTH WEIGHT NEONATES: TIME TRENDS OVER 20 YEARS}

doi:10.1136/archdischild-2012-302724.1077

'MT Moral, ${ }^{2,3}$ L Gutierrez, ${ }^{4} \mathrm{D}$ Coca, ${ }^{1} \mathrm{MJ}$ Torres, ${ }^{2,5} \mathrm{~J}$ De La Cruz, ${ }^{1} \mathrm{CR}$ Pallás. ${ }^{1}$ NeonatologySAMID; ${ }^{2}$ Clinical Research, Hospital Universitario 12 de Octubre; ${ }^{3}$ Preventive Medicine, HUC San Carlos; ${ }^{4}$ Radiology; ${ }^{5}$ CIBERESP-IMAS12, Hospital Universitario 12 de Octubre, Madrid, Spain

Background and Aim Cranial ultrasound (CUS) routine screening examinations have been routinely performed in preterm neonates for decades. The aim of this study is to explore changes over time in the occurrence of severe neonatal brain injury as a function of neonate characteristics and clinical practice features.

Methods Inborn neonates from a single centre (Hosp 12 Octubre, Madrid) in years 1991-2010 with birthweight (BW) <1500g entered a CUS protocol. Brain injury was defined after its full evolution. Three types of severe brain lesions were described: intraventricular haemorrhage (IVH) grade III, parenchymal lesion (PL) III-IV, and ventriculomegaly III. Time trends for brain injuries, neonate characteristics and clinical practice features were explored with generalized estimation equations for logistic regression.

Results 1574 neonates were included in the 20 year study period 1298 (82.5\%) survived the NICU, 33\% BW <1000g. From 1991-2006, severe IVH was in the range $4-6 \%$ overall; between $3-4 \%$ in survivors and $17-31 \%$ in non-survivors, odds ratio $7(4-11)$. Severe parenchymal lesions dropped from 8-9\% to 3-4\% overall after year 1998,
OR 0.35(0.14-0.85); it was much higher in non-survivors (initially, $40 \%$ ) than in survivors (down to $3 \%$ in $1999-2006$, OR 5(3-8)). In the last 4 year period, a significant increase in severe HIV/PL was documented, particularly in $\mathrm{BW}<1000 \mathrm{~g}$, parallel to a further survival increase.

Conclusion No different time trends for severe brain injury were observed in survivors and non-survivors. Variations in characteristics (e.g. increased survival at lower gestational age) may be related with changes in neonatal brain injury outcomes over time.

\section{REGIONAL OXYGEN SATURATION DURING PRETERM NEONATAL TRANSITION}

doi:10.1136/archdischild-2012-302724.1078

${ }^{1} \mathrm{M}$ Pocivalnik, ${ }^{1} \mathrm{C}$ Binder, 'B Urlesberger, 'E Kratky, ${ }^{2} \mathrm{~A}$ Avian, 'W Müller, 'G Pichler. ${ }^{1}$ Department of Pediatrics, Division of Neonatology, Medical University Graz; ${ }^{2}$ Institute for Medical Informatics, Statistics and Information, Medical University Graz, Graz, Austria

Objective To evaluate arterial and regional tissue oxygen saturation during immediate transition after birth in late preterm infants with and without respiratory support.

Methods Prospective observational study. Using nearinfrared spectroscopy, changes in regional oxygen saturation of the brain (rSO2brain) and peripheral preductal tissue (rSO2pre) were measured during the first 15 minutes of life after elective caesarean section. SpO2 and heart rate were measured continuously. Fractiona tissue oxygen extraction (FTOE) was calculated for all regions. According to need for respiratory support two groups were formed, respiratory support (RS) group and normal transition (NT) group (without need for respiratory support). Positive pressure ventilation was applied with the T-piece resuscitator, oxygen was applied according to $\mathrm{SpO} 2$ values. The Florian Respiratory Function Monitor was used to monitor all ventilation parameter. All was recorded including videography.

Results 49 preterm infants fulfilled entry criteria, 7 had to be excluded because need for intubation. Of the remaining infants, 21 formed NT group, and 21 RS group. Heartrate showed comparable courses in both groups. SpO2, rSO2brain and rSO2pre values showed significantly different courses in the two groups, always the NT group showing higher values. FTOE course was significantly different, showing a delayed decrease in RS group.

Conclusion First systematic analysis of behaviour of regional and arterial oxygen saturation in late preterm infants with and without need for respiratory support. Infants with respiratory support showed significantly different oxygen saturation values, all were decreased compared to a normal transition group.

\section{SLEEP-WAKE CYCLING IN HEALTHY TERM NEWBORN INFANTS IN THE IMMEDIATE POSTNATAL PERIOD}

doi:10.1136/archdischild-2012-302724.1079

1,2| Korotchikova, 1,2CA Ryan, 1,2GB Boylan. 'Neonatal Unit/Neonatal Brain Research Group, Cork University Maternity Hospital; ${ }^{2}$ Paediatrics and Child Health, University College Cork, Cork, Ireland

Background and Aims As sleep-wake cycling (SWCing) of healthy infants within hours of birth has not been quantified with conventional EEG monitoring, in this study we examine the SWC composition of healthy term infants in the immediate postnatal period using EEG, and investigate factors that might influence neonatal sleep.

Methods Multichannel video-EEG was recorded for up to 2.5 hours in healthy term infants soon after birth. The total amount and percentages of sleep states (SSs) were calculated for each infant. Parametric/non-parametric statistical testing was used to test the influence of maternal and infant-related factors on SSs. 